\title{
STRUCTURAL PRE-ANALYSIS THROUGH IMPLEMENTING REVIT STRUCTURES AND ROBOT TO DEVELOP AND COMPARE ENGINEERED TIMBER STRUCTURAL FAMILIES
}

\author{
LIVIO LAMARTINA \& JOAO SA \\ Architectural Technology and Construction Management, Copenhagen School of Design \& Technology, Denmark
}

\begin{abstract}
The structural pre-analysis is a teaching technique based on the implementation of BIM for structural design and finds its theoretical didactical background on the theory of didactical situations and designs intended learning outcomes. It provides a problem-based learning approach and wish to shape future professional handling techniques in constant development. The implementation is focused on two main aspects combined together: structural performance (sizing for load bearing capacity) and the sustainability of engineered timber structures. The aim is to test the effect of structural loads and verify both performance compliance (overall dimension and ratio of a load bearing section) and sustainability $\left(\mathrm{CO}_{2}\right.$ footprint in relation of the material involved in the lean processes) combining BIM tools and fine elements analysis software. The system implements Autodesk ${ }^{\mathbb{B}}$ Revit $^{\mathbb{R}}$ Structure with the bidirectional interoperability with Autodesk ${ }^{\circledR}$ Robot $^{\mathrm{TM}}$ Structural Analysis Professional to develop structure generated by modified, built-in families according to manufacturer's mechanical properties. Models can be compared within the analytical results of structural performance and $\mathrm{CO}_{2}$ footprint. The competence of the students can be enhanced in a way to make them able to develop a 3D virtual structural model from a low to high level of development, to choose correctly pre-dimensioned elements from manufacturer's catalogs or, eventually, to dimension realistically a load bearing engineered timber element, always with an eye on sustainability. This teaching procedure provides a second final set of student's outcomes: shop drawings, generated and drawn with a high level of accuracy. This is in conjunction with problem-owners from the international construction and building industry. In this way, the number of iterations during the design phases can be minimized and the role of an architectural technologist can be more interlocked with both structural engineering and production procedures.
\end{abstract}

Keywords: PBL, structural modeling, revit structure, robot, engineered timber, sustainability.

\section{INTRODUCTION}

The implementation of Autodesk ${ }^{\circledR}$ Revit $^{\circledR}$ Structure software (thereafter called Revit Structure) and Autodesk ${ }^{\circledR}$ Robot $^{\mathrm{TM}}$ Structural Analysis Professional software (thereafter called Robot) - both products have free licenses for both students and teachers of Copenhagen School of Design \& Technology (hereafter called KEA) - started at KEA's Bachelor in Architectural Technology and Constructoin Management Intenational (thereafter called BATCM INT) in August 2016 within L. Lamartina's lectures.

The main scope was to use BIM tools as didactic support within Structural Engineering lecturing and class consulting.

Semester after semester it has been developed, according a defined 2nd, 3rd, 4th semester's knowledge and skills-based progression able to design a didactic methodology facilitating student's gaining competences incrementally. The cooperation with teachers from different subjects such as Architecture, Structure, Materials, Structural Engineering, Services, Process and Planning, developed step by step according to coordinated lecture plans. The cooperation with Joao Sa started in January 2019.

Problem based learning (thereafter called PBL) begins when students are confronted with an open-ended, structured, authentic (real-world) problem and work in teams to identify 
learning needs and develop a viable solution, with instructors acting as facilitators rather than primary sources of information. In other words, PBL is an instructional method where relevant problems are introduced at the beginning of the instruction cycle and used to provide the context and motivation for the learning that follows. It is always active and usually (but not necessarily) collaborative (instructional method in which students work together in small groups toward a common goal) or cooperative (structured form of group work where students pursue common goals while being assessed individually).

The wish is to shape future professional handling techniques in constant develop. In this way, BIM facilitates and boosts this workflow. In the specific BIM for structural design belongs totally to the PBL approach.

Architectural technologists typically start the design process by interpreting architectural drawings, creating design documentation, and Structural Engineers creating various analytical models. The creative part of this didactical research has its origin in the wish to merge the above mention professionals in one, acting, at least, from the early design stages.

This can be possible only if Architectural Technologist students can achieve Intended Learning Outcomes in BIM for structural engineering. This Didactical Situation, fits well with the professional profiles described in Chapter 3.

As soon as the student generates an Analytical Model this must be consistently coordinated with respect to general framing size and layout, material and section properties, and loading. As far as analysis and design is complete structural design documentation can not be completely generated, otherwise need to be reviewed or modified to reflect the most current design.

In case of following review's changes this workflow can be repeated for each iteration of the design process.

Traditionally this procedure can be slow with the risk of lengthening the time.

BIM techniques for structural design can minimize the time wasting during the design iterations in communication, information exchange, 3D modeling already from the early design stages.

In fact, the bidirectional interoperability of Revit Structure and Robot reduces the time needed to create and update multiple analysis models and helps to avoid potential errors resulting from a manual coordination between analysis results and construction documentation; practically helps to make this iterative workflow smoother by facilitating the coordination of design documentation with structural analytical design information.

Moreover, Revit Structure capabilities enable students to enrich the physical model with information such as physical properties, proposed analytical model definition, and expected loads conditions.

This makes the physical model more complete and also play the role of icebreaker in communicating with structural engineers.

For example, in a traditional workflow the designer of load bearing structures (which is not necessarily a Certified Structural Engineer) creates a physical model defining expected relations with its analytical representation, which is a simplification of more detailed, "real" geometry. In this fashion, the designer of load bearing structures must then wait until the certified engineer has completed the structural analysis and design before starting coordination and documentation tasks.

The structural pre-analysis Procedure (Chapter 2) sets some guidelines allowing the designer of load bearing structures to minimize time wasting and design iterations from the early design stages using specific BIM tools and techniques. 


\section{THEORY OF DIDACTICAL SITUATIONS AND PRE-ANALYSIS PROCEDURE}

The theory of didactic situations was developed within the field of the didactics of mathematics and it has found widespread use in this field. Obviously these situations are closely linked to mathematics. In that sense there is no doubt that the theory of didactic situations is almost exclusively a didactic theory for mathematics.

However, the theory uses a conceptual framework that can be used in a more general sense, including an understanding of learning and a basic description of teaching situations that not only bear relevance to the didactics of mathematics, but that are also relevant in the didactics of other subjects, at least the didactics of the technical subjects including BIM.

\subsection{The five phases in the didactic situation}

The modern view of teaching requires that the teacher can provoke the expected adaptation in his or her students by presenting them with a carefully selected series of "problems". These problems must be selected in such a manner that the students can accept them, and they are motivated to act, think and evolve. The students know very well that the problem they are presented with was selected with a view to helping them acquire new knowledge. However, the students also know that this knowledge is completely based in the inner logic of the situation, and that they can construct it without calling on didactic reasoning. Not only can they do this, they must, as it is not until the students have fully acquired this knowledge that they are able to use it in situations outside of the learning context and where there is no guidance. This called an adidactic situation. Any specific "piece of knowledge" can be characterised by one or more adidactic situations that secure the meaning of this knowledge [1].

An important element of the theory of didactic situations is the description of the various phases of the didactic game. The didactic game refers to the interaction between the student and the didactic environment. The five phases in the didactic game are:

- Devolution: the teacher passes a "didactic environment" to the students

- Action: the students work in the environment

- Formulation: the students express themselves and create hypotheses about the solution to the assignment, either independently or in groups.

- Validation: the students test their hypotheses together or with the teacher.

- Institutionalization: the teacher relates the work being carried out in the environment to the general themes of the subject, typically through a dialogue with the students.

\subsection{Workflow based on didactical situation in the standard 4th semester at KEA}

Below is a recommended workflow for concurrent structural documentation, design, and analysis based on coordinated subjects lecture plans (Structural Engineering, Structure, Architecture, Materials, BIM, Process and Planning, Services, Communication) lecturing in the standard 4th semester at KEA.

Based on this, the phases of the didactic game through implementing Revit structure and preanalysis procedure in the project-based teaching in the bullet list below.

- Devolution: the students, working in group, create a structural model based on an existing architectural model or existing architectural 2D layouts.

- Action: pre-analysis technically starts here. The student eventually in mutual coordination with the teacher adjusts material and profile definitions by rules of thumb, and adjusts the analytical model proposed by Revit software. 
According Eurocodes, the student implements loads and load combinations that can be used to perform static and wind load calculation in Robot, as well as preliminary or load takedown analysis available with Revit Structure Extensions. The results can be used within design and code checking procedures.

Through interoperability Revit Structure-Robot, Revit Structure's analytical model is transformed by Robot in a 3D Fine Element Model, so the Robot's calculation engine performs structural analyses from Fine Elements; subsequently these analytical results are sent back to Revit Structure and displayed.

The students exploits interoperability under teacher's engineering control.

- Formulation: based on analysis outcomes, the student may make decisions that need coordination with other designers (and perhaps ultimately the architect as well).

- Validation: based on Revit Structure - Robot interoperability, the engineer (teacher) first checks the results then and alerts the designer (student). The student adjusts section sizes and properties, based on information received from Robot Structural Analysis Professional or other code checking applications linked with Revit software. The student updates the analytical model with recommended changes.

The student, using Revit software, reviews teacher's recommended changes to the analytical model. In collaboration with the engineer (teacher), the designer (student) accepts or rejects the proposed changes to the analytical model and the Revit software automatically adjusts the physical model accordingly.

- Institutionalization: this iterative collaboration (consultancy classes) between the designer and engineer repeats as necessary. In study situations, this phase requires a tight collaboration with the teacher that acts as Structural Engineer.

2.3 The pre-analysis and learning progression

About structural modeling (physical and analytical model) the main Intended Learning Outcome (ILO) is to create a physical model for coordination and documentation, and an associated analytical model for structural analysis.

The students, working in groups, upgrade the 3D model according to Level of Development (LOD) progression; LOD is the degree to which the element's geometry and attached information has been thought through; the degree to which project team members may rely on the information when using the model [2].

In the 10 steps of progression, the pre-analysis phase is supposed to be from step 1 to 6 :

1. Dimensioning load bearing elements with rules of thumb

2. Generate the first version of the LOD 200 structural model

3. Applying Boundary conditions

4. Applying Load cases

5. Robot structural analysis BIM interoperability for results verification and validation

6. Second iteration on the LOD 200 Structural model

7. Generating LOD 300 model

8. Generating LOD 350 model

9. Generating LOD 400 structural nodes

10. Generating Shop drawings for production and industry 
About structural analysis (bidirectional linking Revit Structure - Robot) groups achieve the following ILOs:

- $\quad$ Generate load plans for multi-storey buildings according to Eurocode 1.

- Conduct structural analysis and export to analysis and design applications with the analytical model while you are creating the physical model in Revit.

- Integrate analysis results into the BIM process and work in an iterative design workflow.

- Compare different solutions in terms of mechanical properties and $\mathrm{CO}_{2}$ footprint (see paragraphs 4.1 and 4.2).

Different models (both physical and analytical) can be compared through importing both mechanical properties and $\mathrm{CO}_{2}$ footprint of built in and user defined structural families of components.

- Progress Level of Development (LOD) from the LOD 200 pre-analysis to LOD 400 and generate accurate shop drawings.

- Transfer the 3D model for interoperability and documentation with other BIM tools $(4 \mathrm{D}, 5 \mathrm{D})$.

\section{SHAPING PROFESSIONAL}

The aim of the pre-analysis procedure in structural design is to tailor a learning process as close as possible to the building industry practice able to train industry practice-oriented professionals.

Many firms use the Revit Structure model to coordinate their designs with other disciplines. Therefore, structural drafters can begin modeling, documenting using company standards, and coordinating the structure. By starting the design in Revit software, there is both a physical model created for coordination and early documentation, as well as a simplified analytical model. Each model is independently editable but also maintains a consistent relationship to the other.

\subsection{Construction designer of load bearing structures}

According to the last release of the Danish Description of services for Building and Landscape 2018, plan of work for Architects and Engineers, the construction designer of load-bearing structures is the operator (company), who, under the Building Regulations, is responsible for collating and coordinating the structural documentation for the structure [3]. Briefly, a professional handling this process is supposed to enhance his competence about structural documentation through:

- Collecting and coordinating the documentation for the entire structure, according with the provisions of the Danish Building Regulations.

- Assisting the consultants and any design contractors in assessing the consequences of any revised project for the supporting structures of the structure and updates the documentation of the structure.

- Coordinating changes and optimizing the 3D BIM structural model through maintaining mechanical properties (physical and material consistency) at all design stages.

- Assisting the construction management and supervision in assessing the consequences of any alterations to the supporting structures during the construction phase. 


\subsection{Architectural technologist}

In regard to Architectural Technology and Construction Management, at Copenhagen School of Design \& Technology, our students deal with a learning approach which shapes these competences:

- Choose manufacturer's products and from the early stages of the design phases.

- Optimize the lean process.

- Make smart decisions: reuse design intent models to make planning and preconstruction decisions earlier.

- $\quad$ Align construction modeling efforts for downstream uses.

- Use Revit models for better communication: to coordinate information to improve office-to-field efficiency, quality assurance, and quality control, collision control.

- Increase construction site layout productivity.

So, it would seem that the education of an Architectural Technologist covers widely the competences related with construction designers of load beating structures from Description of services for Building and Landscape 2018.

4 STUDY CASE: DEVELOPING ENGINEERED TIMBER STRUCTURAL FAMILIES KEA's BATCM INT 4th semester project is given as an example of implementation and develop of load bearing engineered timber structural elements. As study case, 4th semester students are engaged with a multistory office building whose load bearing system is supposed to be made with engineered timber elements (Fig. 1).

The pedagogical choice of develop engineered timber load bearing elements is related to the wish to tailor a didactic approach close to the use of innovative, environmentally friendly building materials. In fact, across the construction landscape, massive wood products from several manufacturers (f. ex. Stora Enso) such as cross-laminated timber (CLT), laminated veneer lumber (LVL) and glulam are being used in both everyday and award-winning applications. Massive wooden materials can play an essential role in building sustainable urban areas for the future.

Implementing engineered timber in the semester project supported by BIM techniques gives the students some advantages that in short are:

- Design without sacrificing structural performances or requirements.

- Quicker construction for time and cost savings.

- Improved thermal insulation and acoustic performance.

- High sustainability credentials for a lower carbon footprint.

The students, working in groups, develop the building's structural load bearing system following the 10 steps shown in paragraph 2.3 .

The pre-analysis phase, in terms of relevant results for overall load bearing system dimensioning are shown in Fig. 2.

The follow up outcomes according to steps 7 to 10 (cfr. 10 steps in paragraph 2.3) are shown in Fig. 3. 

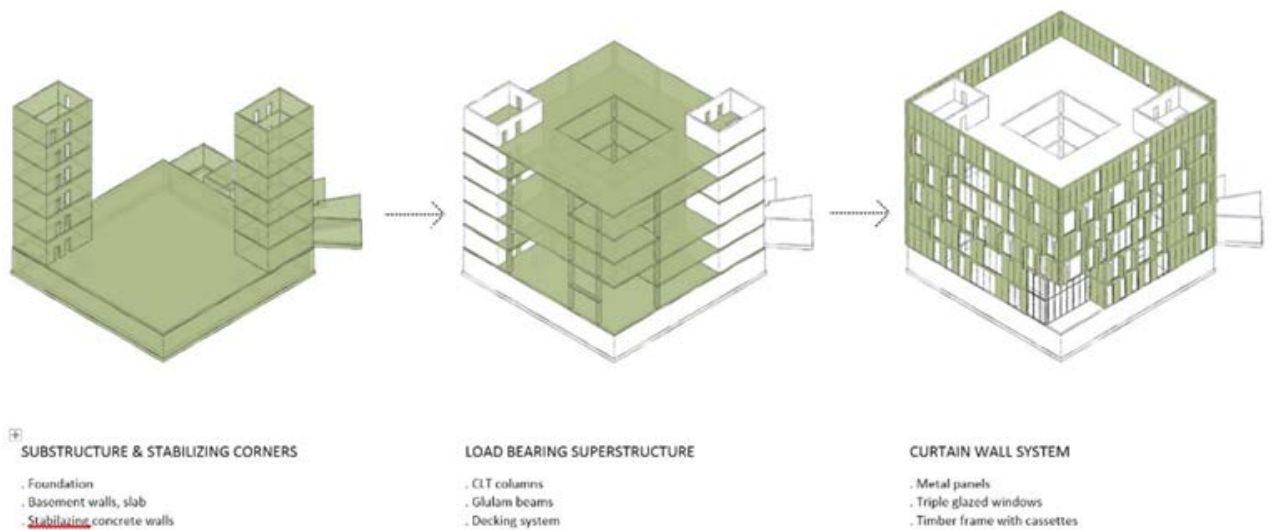

CURTAIN WALL SYSTEM

Metal panels Triple glazed windows

Figure 1: Fourth Semester project, Spring 2019 - structural and curtain wall system.

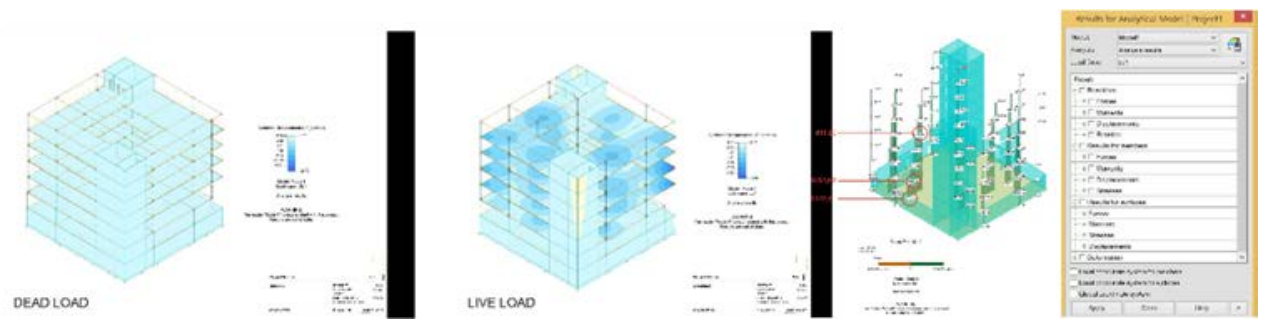

Figure 2: Results for overall load bearing system.

BATCM 4J

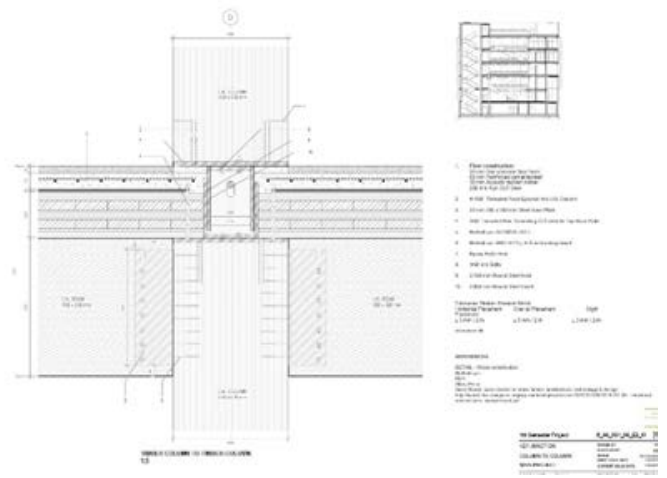

Figure 3: From LOD 300 model and detailing. 
4.1 Engineered timber structural families and user defined material properties

Building a developed 3D structural model, with precision engineered industrialized wood materials embodied in virtual load bearing elements allow the students to push beyond Revit Structure's perceived boundaries.

This is a crucial point in the workflow because it requires accuracy in the model making to avoid potential issues in the analysis.

Elements in Revit are referred to as families. The family contains the geometric definition of the element and the parameters used by the element. Each instance of an element is defined and controlled by the family.

Built in Revit structural families need to be checked and modified to better emulate the real mechanical properties of the material properties of the manufacturer reference component.

Not define physical mechanical properties can give issues and errors when the analytical model is sent to Robot for calculation and interoperability. In this way the students develop skill in Parametric Modeling.

\subsubsection{Parametric modeling}

Parametric Modeling refers to the relationships among all elements in a project that enable the coordination and change management that Revit provides. These relationships are created either automatically by the software or by the user. Revit immediately determines what is affected by changes and reflects those changes to any affected elements. A fundamental characteristic of Revit is the ability to coordinate changes and maintain consistency at all times.

In this way the students must set up manually the mechanical properties of the family's material and so deepen the knowledge about the elements available in the market through an in-depth study of the manufacturer's technical catalogs (Fig. 4).

Pedagogically, correcting structural Revit's families mechanical properties, enhances student's competence through enhancing software's skills and knowledge of construction's materials (Fig. 5).

In Revit structure the properties that define a material are organized into assets. Assets are groups of properties that control certain characteristics or behaviors of an object.

Revit uses the following asset types to define materials:

- Identity - These properties provide general information about the material, such as a description, manufacturer, and cost data.

- Graphics - These properties control how the material looks in non-rendered views.

- Appearance - These properties control how the material looks in rendered views, Realistic views, or Ray Trace views.

- $\quad$ Physical - These properties are used for structural analysis.

- Thermal - These properties are used for energy analysis.

Some manufacturers enable the download of BIM objects libraries. These objects on the one hand allow the student to efficiently imported during the modeling but in most cases they are not true structural elements which don't have a consistent analytical model related withtodesk Robot. 


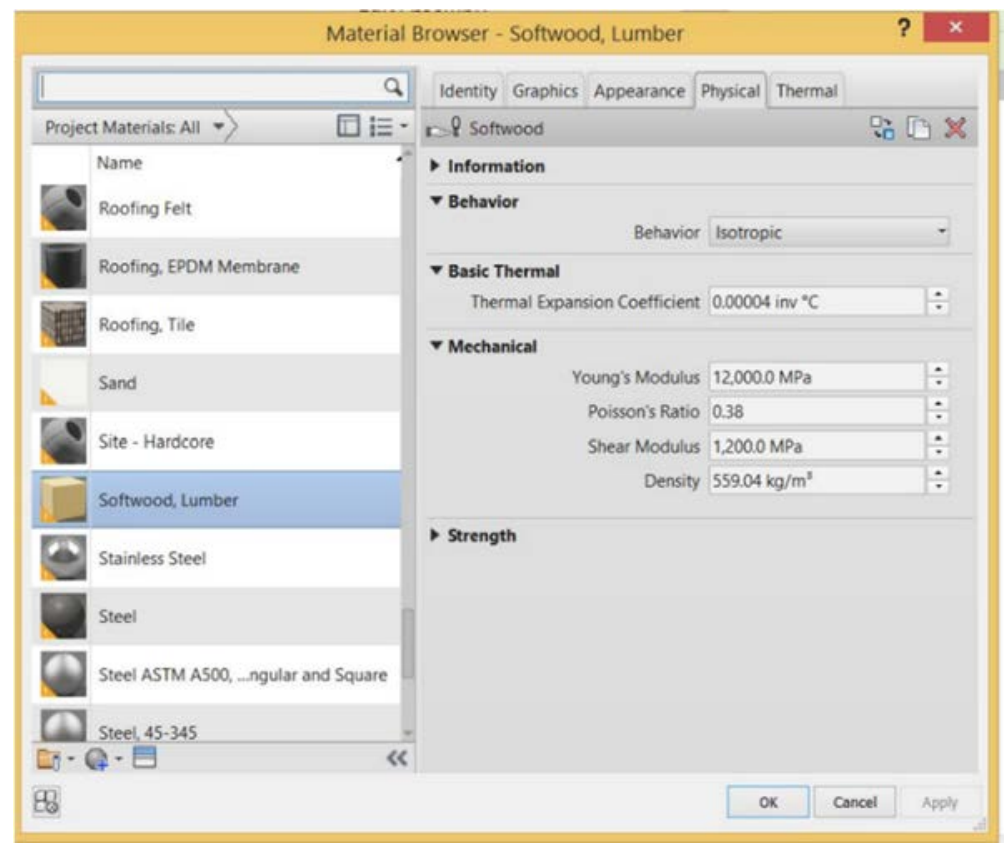

Figure 4: Screenshot of physical properties settings.

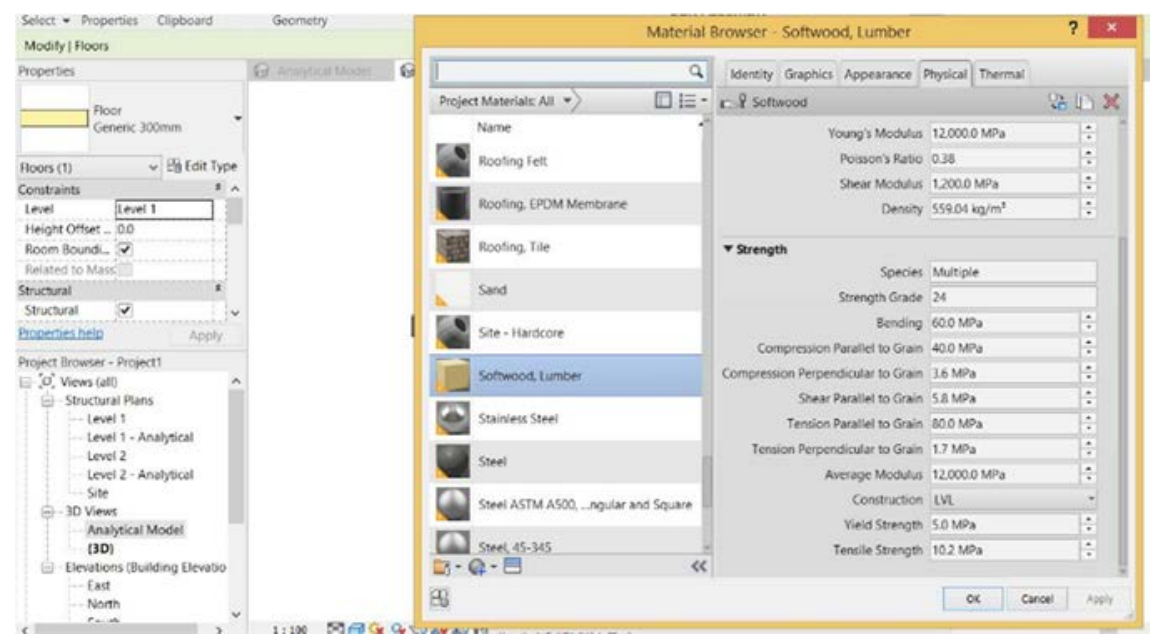

Figure 5: Revit Structure screenshot of user defined mechanical settings for an LVL floor.

4.1.2 Engineered timber structural floors

CLT and LVL Revit Structure built in super structural frame elements (Columns and Beams) are available by default and work with Robot interoperability, though there is no built-in default structural engineered timber floor element. 
However, in Revit Structure, it is possible to obtain engineered timber structural floors, starting from a generic floor redefining material (soft lumber) and physical settings according to manufacturer's declared mechanical properties.

\subsection{Sustainability}

Within KEA's BATCM INT 4th semester teaching team, the didactic supports massive wooden structural materials (CLT and LVL) and we believe that they can play an essential role in building sustainable urban areas for the future.

It's no wonder that engineered timber is a construction material from an industrialized process mostly based on renewable raw materials and sustainable forest management, which contribute to responsible consumption and resource use. The use of renewable materials enables us to contribute towards a low-carbon economy. Trees in sustainably managed forests absorb carbon dioxide $\left(\mathrm{CO}_{2}\right)$ from the atmosphere and together with wood-based products act as carbon storage [4].

Pedagogically, using BIM tools as a support of structural engineering lecturing and consulting cooperates in shaping students - indeed as future professionals - with a design approach conscious of environmental issues.

\subsubsection{Tables for embodied $\mathrm{CO}_{2}$ calculation and comparative template}

$3 \mathrm{D}$ model can be developed also with embodied parametric formulas for embodied $\mathrm{CO}_{2}$ volumes calculation.

The content of this and next paragraph reports a didactic attempt of generating tables for $\mathrm{CO}_{2}$ calculation with the aim to bring to the students a BIM related tool through a procedure which allows to produce documentation for systematic comparative building component's analyses including environmental sustainability facts.

According to KEA'S BATCM INT cross disciplinary BIM-Structural Engineering continuing teaching development, the aim of this experimental exercise is to make a student's mandatory deliverable assignment to be presented at the exam about $\mathrm{CO}_{2}$ embodied in the structural load bearing system.

In the following is reported the first iteration of a table for $\mathrm{CO}_{2}$ footprint calculation inspired by Greenhouse gas protocol calculation tool [5].

In the specific the values of embodied carbon for the materials composing the model, the source is Inventory of Carbon and Energy (ICE) provided by University of Bath Sustainable Energy Research Team [6].

As example for computing embodied $\mathrm{CO}_{2}$ is used a $3 \mathrm{D}$ model of a small structural frame made by massive timber elements (GLT columns, LVL beams, CLT floor) and four footing concrete pads (Fig. 6).

Wood stores carbon and its $\mathrm{CO}_{2}$ footprint is negative (circa - $1000 \mathrm{Kg} / \mathrm{m} 3$ ) [7]. Eventually, when wooden building elements are recycled or reused, the carbon storage is also extended.

About reinforced concrete class 35 (including embedded reinforcement with a ratio of $30 \mathrm{~kg}$ of steel per cubic meter of concrete) the average value of embodied $\mathrm{CO}_{2}$ is $+402 \mathrm{~kg} / \mathrm{m}^{3}$. In this fashion, implementing Revit Structure and developing engineered timber structural families in the semester project, allows the students to generate tables for $\mathrm{CO}_{2}$ footprint calculation using ICE data as reference.

Didactically this outcome facilitates the understanding of the environmental footprint of a timber structural element in comparison, for instance, with a concrete foundation footing element (Tables 1 and 2). 
Table 1: Displayed formula for an embodied $\mathrm{CO}_{2}$ calculation.

\begin{tabular}{|c|c|c|c|c|}
\hline Family Types & & & & $x$ \\
\hline Type name: $\quad 127 \times 140$ & & & $\sim$ 는 & 团 好 \\
\hline Search parameters & & & & Q \\
\hline Parameter & Value & Formula & & Lock \\
\hline Materials and Finishes & & & & 2 \\
\hline Structural Material (default) & Softwood, Lumber & ]$=$ & & \\
\hline Dimensions & & & & 2 \\
\hline b & 200.0 & $=$ & & $\square$ \\
\hline d & 400.0 & $=$ & & घ \\
\hline h (report) & 2500.0 & $=$ & & \\
\hline Green Building Properties & & & & $\therefore$ \\
\hline $\mathrm{CO} 2 \mathrm{FP}$ (defoult) & $-200,000000$ & $=\left(\left(b^{*} d^{*} h\right) / 1 m^{2}\right) *$ Specific CO2 FP & & \\
\hline Specific CO2 FP & -1000.000000 & $=-1000$ & & \\
\hline
\end{tabular}

Table 2: $\mathrm{CO}_{2}$ footprint schedule generated in Revit Structure.

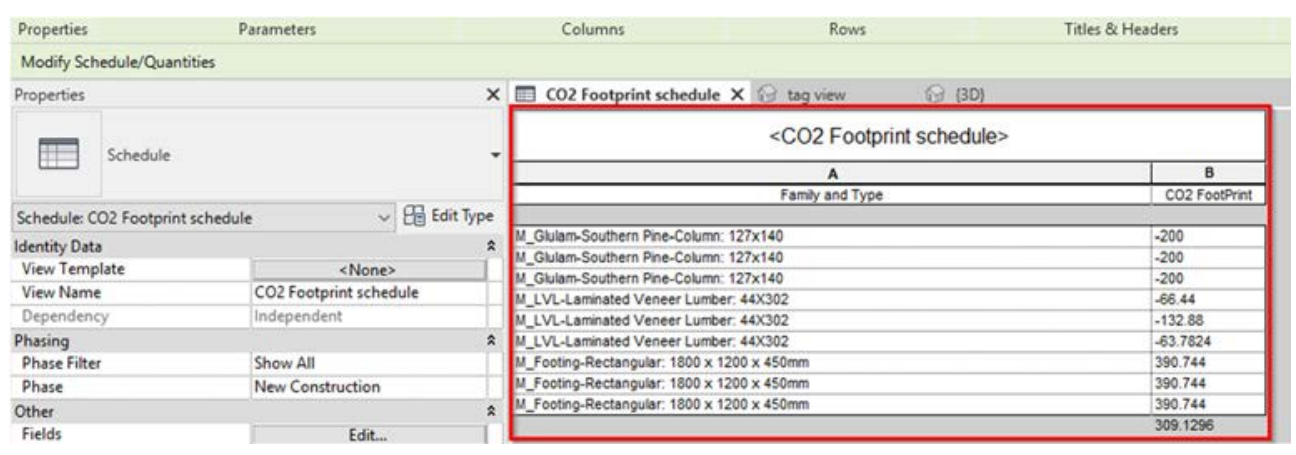

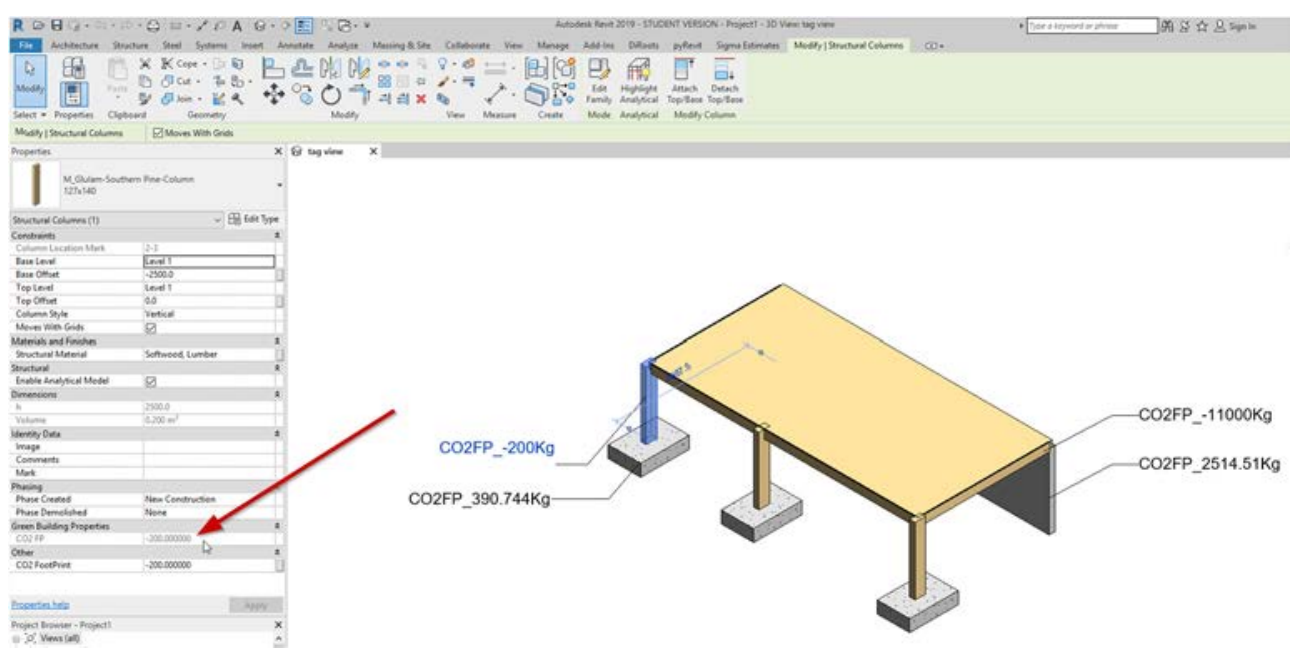

Figure 6: Screenshot of a 3D model associated with the table for $\mathrm{CO}_{2}$ footprint calculation. 
Table 3: Template for structural and sustainability comparative analysis.

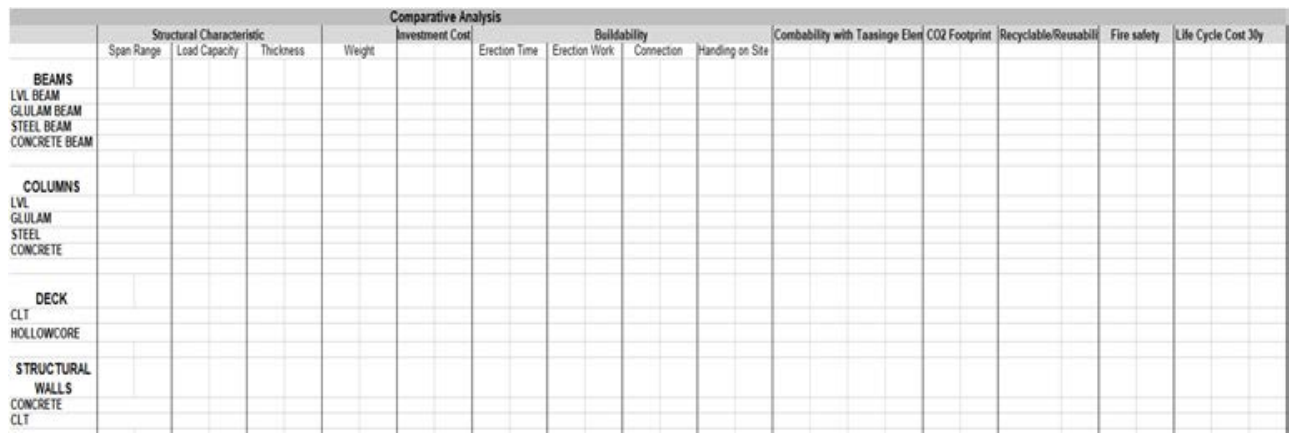

\subsubsection{Comparative analysis}

Students run comparative analysis with the target to have a tool able to give back the best solution according to the design scenario they deal with.

The template they work is a matrix which relates several super structural load bearing components (beams, columns, deck elements, walls made with different materials) in a way to list their main attributes in one template able to give back a general overview (see the template in Table 3).

Some of the attributes are merely determined by numbers but some of them are subjective related with their own capability and competence in evaluating and validating design solution.

The numerical attributes are:

- Structural: Span Range, Load Capacity, Thickness, Weight.

- $\mathrm{CO}_{2}$ Footprint: value from Revit Structure tables.

- Fire safety class: from manufacturer's specification.

- Investment Cost: value from 4D and 5D integration.

- Life Cycle Cost 30 Years: value from 4D and 5D integration.

The subjective attributes are:

- Buildability.

- Combability with other structural elements.

- Recyclability/Reusability.

\section{CONCLUSION}

Structural pre-analysis through implementing Revit Structure and Robot to develop and compare engineered timber structural families embodies new knowledge and practices from industry into the teaching [8]-[10].

The implementation needs to be constantly updated with advances in digital BIM design tools such as construction material properties, challenging so building culture enabling sustainable, informed and materially smart design solutions.

All of this is setting a teaching cross disciplinary template enabling information exchange between different teaching subjects in school (i.e. Structural Engineering, Structures, Material, Architecture, Process and Planning, Communication) and professional disciplines out in the building industry. 


\subsection{Streamlined and productive design process}

Based on the original hypothesis about how to make smarter decision trough upgrading and compare structural solutions in terms of load bearing performances and $\mathrm{CO}_{2}$ footprint, the pre-analysis procedure can minimize the iterations from the early design stages.

In this fashion, the students are facilitated in structuring a more streamlined and productive design process, performing more analysis to help find the best structural design option, and a better understanding of design intent yielding less errors and omissions.

In summary student's competences are enhanced in:

- $\quad$ Making smarter decisions: reuse design intent models to make planning and preconstruction decisions earlier.

- Choosing manufacturer's products and from the early stages of the design phases.

- Aligning construction modeling efforts for downstream uses.

- Coordinating changes and maintaining mechanical properties (physical and material consistency) at all times.

- Optimizing the BIM structural modeling and the workflow related with.

5.2 What's systematic: inductive teaching and theory of didactical situation (TDS)

Teaching structural engineering implementing Revit structure and Robot into the semester's projects, through TDS and inductive teaching brings both students and teacher in a continuous follow-up of processes and results.

The bidirectional link between Revit software and Robot Structural Analysis Professional helps make the exchange of structural analytical information smoother. So, it facilitates the general understanding of structural engineering learning.

The link enables students to add analysis-related information to the Revit model, use that model (and information) directly for analysis in Robot Structural Analysis Professional, and then update the Revit model based on the analysis results.

This systematic exchange of information respects and preserves the information defined in both software solutions.

Within preanalysis procedure, project based teaching and inductive teaching, students achieve Intended learning outcomes (ILOs), and develop skills in 3D structural modeling.

\subsection{Margins of unpredictability}

The presumption of the expected results in achieving learning outcomes implementing didactical BIM techniques in classes and consultancy sets can be affected by interoperability issues and general student's engagement.

\subsubsection{About Interoperability issues}

- Design issues: most of the times, hasty students generate inconsistent 3D analytical models associated with a structural physical model wrongly generated during the design phases.

- Fatal errors: in a study scenario, 3D Structural designing of a multi-storey building need performing computers and could be critical for students working with slow computers. For example, in school situation, is recommended to work with KEA's Virtual Design Construction laboratory instead of using private Laptop. This can avoid computer's fatal errors during both design set and interoperability. 
- Parametrical design Accuracy of material properties: both Physical and Mechanical properties of the built in Revit Families must be modified accordingly with manufacturers declared structural performances. Otherwise a lack of coordination can distort the results of both static calculation and sustainability analysis. The target of parametrical design Accuracy must match with manufacturers data.

\subsubsection{About general student's engagement}

Preanalysis and 3D structural design is a turning point in the semester progression; as long as the groups are focused and skilled it is possible to achieve professional results. The group itself can become an accelerator or a burden to achieve learning outcomes, hand in deliverables and finally perform good exam.

Issues - such as slow work or delay in the hand ins - can be generated by an uncoordinated workflow of the group members in both communication and technical effort.

Group issues due to lack of communication with the teacher and between group members can really afflict the overall semester performance and exam, as well as Lackness of knowledge in interpreting the analytical results: some students are more resilient in understanding the physics behind the structural analytical results; so they risk not to really manage this BIM procedure.

\subsection{What's transferable}

Implementing Revit Structure and developing Engineered Timber Structural families combines:

- Growth of student's knowledge and skills through ILOs firmly related with BIM procedures.

- Coordination of design information and construction documentation by using Revit models for better communication; coordinating information to improve office-to-field efficiency, quality assurance, and quality control.

- Construction site layout productivity in terms of time scheduling (4D) and costs (5D), through interoperability with construction management BIM tools.

- Strengthen cooperation for constant develop and follow up with professional from building industry through the problem ownership: finding external cooperation with building industry professional and offices bringing authentic real-world problems about BIM for structural design.

In summary, this implementation:

- Supports the constant upgrading of a teacher's competence, know how and building industry networking.

- Enhances opportunities to broaden student's horizons: Architectural Technologist can play as a multirole professional. Contemporary broads internship opportunities, can increase the learning curve, facilitate in being problem solvers.

\section{ACKNOWLEDGEMENTS}

- Anne Ambrosy, Elena Cucchi, Rodrigo Jose Cid, Tim Nordahl, Valts Kasparans KEA BATCM INT 4th semester students Spring 2019 for sharing the images of their structural report in Figs 1, 2 and 3.

- Claudio Spaziani Testa, KEA BATCM INT Head of the Programme, for suggestions on professional profiles reported in Chapter 3. 


\section{REFERENCES}

[1] Brousseau, G., Theory of Diactical Situations in Mathematics, eds., trans. N. Balacheff, M. Cooper, R. Sutherland \& V. Warfield, Dordrecht: Kluwer Academic Publishers, p. 30, 1997.

[2] Level of Development Specification, BIM Forum 2016, pp. 11, Online. www.bimforum.org/lod.

[3] The Danish Association of Architectural Firms, The Danish Association of Consulting Engineers (FRI). Description of Services for Building and Landscape, p. 9, Online. https://frinet.sharepoint.com/Aftaleudvalget/YB generelt/YBL 2018.docx.

[4] storaenso. Massive Wood Construction. Online. https://www.storaenso.com/en/products/wood-products/massive-wood-construction.

[5] Greenhouse Gas Protocol. About Greenhouse Gas Protocol, Online. https://ghgprotocol.org/calculation-tools.

[6] University of Bath ICE (Inventory of Carbon and Energy), Online. http://wiki.bath.ac.uk/display/ICE/Home+Page;jsessionid=2358DA949145979F0F88 65783188344D.

[7] Stora Enso. http://www.clt.info/ecological-balance-sheet-clt-holzforschung-austria/.

[8] Pomares, J.C., Baeza, F.J., Varona, F.B. \& Bru, D., BIM Implementation for Structural Design Courses in Civil Engineering, WIT Press, p. 79, 2017.

[9] Mahdjoubi, L., Brebbia, C.A. \& Laing, R., Colec. Building Information Modelling (BIM) in Design, Construction and Operations, WIT Press, 2015.

[10] Pomares, J.C., Baeza, F.J., Varona, F.B. \& Bru, D., Revisión del uso de Building Information Modeling en la Educación Superior relacionada con la arquitectura, ingeniería y construcción, Editorial Octaedro, pp. 991-1001, 2017. 